\title{
Prevalence of PCOD in Patients Visiting OPD of Karuna Medical College
}

\author{
Harrini K.․․ Renuka J. ${ }^{2}$, Sajitha C.K. ${ }^{3}$, Jamila Hameed ${ }^{4}$, Yanisha Abdul Kareem ${ }^{5}$ \\ ${ }^{1}$ Department of Obstetrics and Gynaecology, Karuna Medical College, Palakkad, Kerala, India. \\ 2Department of Obstetrics and Gynaecology, Karuna Medical College, Palakkad, Kerala, India. \\ 32Department of Obstetrics and Gynaecology, Karuna Medical College, Palakkad, Kerala, India. \\ ${ }^{4}$ Department of Obstetrics and Gynaecology, Karuna Medical College, Palakkad, Kerala, India. \\ ${ }^{5}$ Department of Obstetrics and Gynaecology, Karuna Medical College, Palakkad, Kerala, India.
}

\section{ABSTRACT}

\section{BACKGROUND}

The incidence of polycystic ovary syndrome is found to be very common in patients attending gynaecology department. The incidence is growing rapidly among young adults. PCOS leads to multiple complications. A study of 120 women with PCOS was done in Kerala in India in order to analyse the prevalence, clinical features and impact on life.

\section{METHODS}

Case records of women who were diagnosed as Polycystic Ovarian Disease who attended the gynaecology OPD in Karuna Medical College Hospital in Kerala were randomly selected. Women included in the study were in the age group of $14-30$ years. The sample size selected was 120 . The study was carried out for a period of 12 months in our hospital with the help of the records available. The diagnosis was made as per Rotterdam's criteria.

\section{RESULTS}

75 women were unmarried and 45 married. 2 of the women had subclinical hyperandrogenism. 70 women have DHEA increased. FSH: LH ratio > 2:1 seen in 84 women. Testosterone was normal. Anti-Mullerian Hormone was raised in 84 women, indicating hyperandrogenism. Serum Prolactin was normal in most women except in 2 women.

\section{CONCLUSIONS}

PCOD leads to many complications like diabetes, Metabolic $\mathrm{X}$ syndrome, cardiovascular complications, breast cancer, endometrial cancer and depression. Kerala is considered as the diabetic capital in India. There are several risk factors like, family history, lack of exercise, stress, and high carbohydrate diet. A screening programme for PCOD is essential to prevent the sequelae.

\section{KEY WORDS}

Polycystic, Ovary Syndrome, Prevalence, Sequelae, Diabetes
Corresponding Author: Jamila Hameed, D66, Doctors Quarters, Karuna Medical College and Hospital, Palakkad, Kerala, India. E-mail: jamilahameed@gmail.com

DOI: $10.14260 / \mathrm{jemds} / 2020 / 347$

Financial or Other Competing Interests: None.

How to Cite This Article:

Harrini K, Renuka J, Sajitha CK, et al. Prevalence of PCOD in patients visiting OPD of karuna medical college. J. Evolution Med. Dent. Sci. 2020;9(20):1590-1593, DOI: $10.14260 /$ jemds/2020/347

Submission 27-03-2020,

Peer Review 01-05-2020,

Acceptance 04-05-2020,

Published 18-05-2020.

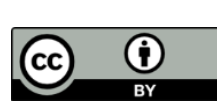




\section{BACKGROUND}

PCOD is a syndrome consisting of amenorrhea, hirsutism, Poly cystic ovary and obesity. It is the most common disorder in the young adults. ${ }^{1}$ It is manifestation of excessive androgen produced by the theca cells of the enlarged ovary. It is characterized by alteration in the LH/ FSH more than 2:1. Normally it is 1:2 as such endometrial hyperplasia occurs leading to endometrial cancer and breast cancer. Due to continuous LH increase, LH surge is lacking leading to an ovulation and this leads to infertility.

As a sequela of anovulation which leads to decrease progesterone the women get menometrorrhagia and finally leads to oligomenorrhea or even amenorrhea. Finally results in multiple cysts in the ovary due to decrease FSH. The excessive theca cells producing androgen under the influence of high LH leads to hirsuitism ${ }^{2}$ due to peripheral conversion of the androgen further to oestrone which leads to hyperlipidaemia and obesity which result in cardiovascular disease.

The hall mark of PCOD is obesity ${ }^{3}$ wherein BMI is $>30$ $\mathrm{Kg} / \mathrm{m}^{2} .^{4}$ The waist to hip ratio more than 0.85 . Due to android obesity hyper insulinemia is associated with PCOD. Hence it results in insulin resistance. This causes increase androgen production by the ovary leads to decrease hepatic SHBG Normally insulin resistance is noted by fasting serum insulin which is $>25 \mathrm{mIU} / \mathrm{ml}$. Serum Fasting Blood sugar and serum fasting insulin is an important factor to be noted.

The insulin resistance ${ }^{5}$ leads to hyper pigmented patches similar to Type 2 D.M. on the skin, in the nape of neck and axilla.

Sometimes under the breast called acanthosis nigricans ${ }^{6}$ which is associated in the acronym HAIR AN syndrome, Hyperandrogenism insulin resistance, acanthosis. PCOD is like a sleeping tiger any time it can wake up causing metabolic X syndrome ${ }^{7}$ in future, which is characterized by-

\section{Any 3 of the following $\mathbf{5}$ Criteria $^{\mathbf{8}}$ are Present}

1. Abdominal obesity $>35$ inches.

2. $\mathrm{HDL}<50 \mathrm{mg} / \mathrm{dL}$

3. Triglycerides $>150 \mathrm{mg} / \mathrm{dL}$

4. $\mathrm{BP}>130 / 85 \mathrm{mmHg}$

5. Fasting Blood sugar level $110-126 \mathrm{mg} / \mathrm{dL}$.

1. There are plenty of hormones which are increased (namely androgen, testosterone, androstenedione, DHEAS)

2. L.H. $>10 \mathrm{IU} / \mathrm{ml}$

3. Oestrone is increased than oestradiol which is least potent.

\section{But Total Free Oestrogen is Increased}

1. Insulin $>10 \mathrm{mIU} / \mathrm{L}$ is noted due to insulinemia and insulin resistance.

2. LDL, Cholesterol and Triglycerides are increased.

3. Some patients have increased prolactin.

The hormones which are decreased are the progesterone because of lack of ovulation FSH is decreased leading to polycystic ovaries.
Serum SHBG decreased and increase in androgen leading to increased testosterone which causes hirsutism. The most important is HDL which is decreased leads to increase in the cardiovascular diseases.

PCOD is multi organ disorder. There is a marked disorder in Hypothalamo - pituitary ovarian axis as such there is a derangement in the hormones leading to organ disorder. This was noted initially by Stein Leventhal and called it as Stein Leventhal Sydrome ${ }^{9}$ in the year 1935.

In 2003, Rotterdam criteria ${ }^{10}$ was set for diagnosis of PCOD. Any two of the 3 following criteria to be present to diagnosis as PCOD.

1. Oligomenorrhea or amenorrhea.

2. Clinical features (hirsutism, acne, alopecia) or biochemical features of hyperandrogenism. Testosterone $70-150 \mathrm{ng} / \mathrm{dL}(200 \mathrm{ng} / \mathrm{dL}$ testosterone secreting ovarian tumours)

3. Polycystic ovary ${ }^{11}$ more than 12 or more cysts, each cyst $2-9 \mathrm{~mm}$ in size in any one ovary or both ovaries with volume more than $10 \mathrm{ml}$.

PCOD is the diagnosis of exclusion. A lot of other cases mimic PCOD wherein hirsutism is present. Hirsutism is the distribution of sexual hair in the female like a male distribution pattern. Ferriman-Gallwey ${ }^{12}$ score between 4-6 indicates hirsutism. It is also noted in adrenal hyperplasia, adrenal tumour, Cushing's disease, hyperprolactinemia, hypothyroidism and masculinising tumour of the ovary. When hirsutism is noted in an aggressive rapid growth, tumour has to be suspected. In such cases virilisation and masculinisation like increase in muscle mass, clitoromegaly, deepening of the voice, frontal balding, acne and breast atrophy are noted. Such changes are rarely seen in PCOD. Hair growth in a female androgen dependent which is from the adrenals and ovary. The free testosterone which is not bound to SHBG is biologically active and causes hirsutism.

\section{METHODS}

A total number of patients 120 who were diagnosed as PCOD on the basis of Rotterdam's criteria attended the gynaecology OPD in Karuna Medical College Hospital were selected by purposive sampling. PCOD was defined as the presence of any two of the three features-

1. Oligo/amenorrhoea: absence of menstruation for 45 days or more and/or $</=8$ menses per year.

2. Clinical hyperandrogenism: Modified Ferriman and Gallway (MFG) score of 6 or higher.

3. Polycystic ovaries: presence of 10 or more cysts, $2-8 \mathrm{~mm}$ in diameter, usually combined with increased ovarian volume of $>10 \mathrm{~cm}$

4. Dense thecal stroma.

The women included in the study belonged to the age group of 14-30 years. The patients complaining of menstrual problems, anxious to conceive, were taken into consideration. As per the Rotterdam's criteria, USG reports, signs and symptoms of hyperandrogenism are included in the inclusion criteria. Ethical committee permission was taken. Hirsutism, BMI, menstrual history and other features of 
hyperandrogenism and insulin resistance were studied. The different aspects of the prevalence of PCOD are studied.

\section{Statistical Analysis}

The data were entered into Microsoft Excel and analyzed using Epi info software for statistical analysis.

\section{RESULTS}

The total number of patients in the study was 120 . The diagnosis was made as per Rotterdam's criteria. Exclusion criteria are congenital adrenal hyperplasia, hyperprolactinemia, thyroid disorder, Cushing syndrome and androgen secreting tumours. PCOD is a common disorder and a diagnosis of exclusion. 75 women were unmarried and 45 married. 2 of the women had subclinical hyperandrogenism. 70 women have DHEA increased. FSH: LH ratio $>2: 1$ seen in 84 women. Testosterone was normal. Testing done only in forty patients. Seventy-five patients were obese. Fifteen patients were overweight and twenty-five had normal weight and five had below normal weight. Diabetes was noted three women. Hypertension was noted in ten women. Impaired glucose tolerance was noted in thirty-six women. Binge eating was noted in eighteen women.

Anti-Mullerian Hormone raised in 84 women, indicates hyperandrogenism. Transvaginal ultrasounds with the probe $8 \mathrm{MHz}$ transducer pick up polycystic ovaries and has shown higher antral count in 60 women. Serum Prolactin was normal in most women except in 2 women (within $40 \mathrm{ng} / \mathrm{ml}$ ) Whenever there is increased prolactin, we must rule out hyperprolactinemia may be due to prolactinoma wherein the patient complains of headache, galactorrhoea, amenorrhoea and visual problems. Whenever it is more than $20 \mathrm{ng} / \mathrm{ml}$ it is considered as hyperprolactinemia. HbA1c was $>7 \%$ in 3 women and the rest had $<6.5 \%$ Higher investigations like coronary artery calcium, carotid intimal media thickness which are done to rule out stroke and myocardial infarction were not performed. There is a wide variation noted in prevalence of PCOD in women based on different criteria we have studied the community-based study in the southern Indian population in Kerala.

\begin{tabular}{|ccc|}
\hline Multiple follicles on USG + oligo & Number & $\%$ \\
Mf on USG + Oligo + Obesity + Acanthosis & 20 & 16.7 \\
Mf + oligo + obesity + acanthosis + hirsutism & 13 & 10.8 \\
Mf + oligo + obesity + hirsutism & 13 & 10.8 \\
Mf + oligo + obesity & 20 & 16.7 \\
Mf + oligo + acanthosis & 20 & 16.7 \\
Mf + oligo + hirsutism & 14 & 11.6 \\
Table 1. Polycystic Ovary Syndrome & \\
\hline
\end{tabular}

\begin{tabular}{|ccc|}
\hline BMI & Frequency & $\mathbf{\%}$ \\
$<18$ & 15 & 12.5 \\
$18-22.9$ & 25 & 20.8 \\
$22.9-25$ & 5 & 4.1 \\
$25-30$ & 34 & 28.3 \\
$30-35$ & 10 & 8.3 \\
$>35$ & 31 & 25.8 \\
Total & $\mathbf{1 2 0}$ & $\mathbf{1 0 0}$ \\
\hline & Table 2. PCOS and BMI \\
\hline
\end{tabular}

\begin{tabular}{|cc|}
\hline Complaint & Present \\
Hirsutism & 90 \\
Oligomenorrhea & 30 \\
Amenorrhoea & 20 \\
Infertility & 15 \\
Acne & 40 \\
Acanthosis nigricans & 20 \\
Baldness & 10 \\
Obesity & 75 \\
\hline Table 3. Clinical Features in PCOS \\
\hline
\end{tabular}

\begin{tabular}{|ccc|}
\hline Age (Years) & Frequency & $\mathbf{\%}$ \\
$14-18$ & 37 & $30.8 \%$ \\
$19-22$ & 32 & $26.6 \%$ \\
$23-26$ & 33 & $27.5 \%$ \\
$27-30$ & 8 & $6.6 \%$ \\
Above 30 & 10 & $8.5 \%$ \\
Total & $\mathbf{1 2 0}$ & $\mathbf{1 0 0}$ \\
\hline Table 4. Prevalence of PCOD in Various Age Groups \\
\hline
\end{tabular}

\begin{tabular}{|ccc|}
\hline Menarche Age (Years) & Frequency & $\mathbf{\%}$ \\
11 & 3 & 2.5 \\
12 & 22 & 18.33 \\
13 & 34 & 28.33 \\
14 & 39 & 32.5 \\
15 & 10 & 8.33 \\
16 & 10 & 8.33 \\
17 & 2 & 1.66 \\
18 & Nil & 0 \\
19 & Nil & 0 \\
Total & $\mathbf{1 2 0}$ & $\mathbf{1 0 0}$ \\
\hline \multicolumn{2}{c}{ Table 5. Incidence of Age of Menarche in PCOD } \\
\hline
\end{tabular}

\section{DISCUSSION}

The exact aetiology of PCOD remains obscure. It has got both environmental and genetic predisposition. Pathophysiology of PCOD is mainly due to disorder in the hypothalamopituitary-ovarian axis. It is a functional disorder as a result there is anovulation and it is a vicious cycle. Whether anovulation is a cause or result in PCOD is obscure. Not a universal diagnostic criterion for diagnosis of PCOD is available. Even obesity is considered as a genetic disorder and not an essential feature of PCOD, but adds on to the pathophysiology. The primary treatment for polycystic ovarian syndrome is to change the lifestyle with medication. The objective of the treatment is to reduce the insulin resistance by insulin sensitizers like Metformin.

To tackle the problem of hirsutism and cosmetic issues like acne, baldness by offering them OCP having least androgen activity like Desogestrel, gestodene, norgestimate. Drospirenone newer progestins are preferred. Epilation methods like waxing, threading and plucking. Electrolysis or laser is preferred but needs repeated visits to the beauty clinic. The drugs which inhibit 5-alpha-reductase namely Finasteride and anti-androgens like Flutamide, Spironolactone and Cyproterone acetate are useful. The eflornithine hydrochloride cream is recommended.

The lifestyle modification is important as PCOD is associated with obesity and overweight. The diet plan is almost similar to the diabetic patient. The meal consists of fresh vegetables, fruits and whole grain. The micronutrients and vitamins are given importance. The treatment is based on the complaints and the scenario. In case of infertility it can be tackled with Clomiphene citrate, ovulation induction drugs. But one has to be careful about ovarian hyperstimulation syndrome. So, a follow up of the follicular study by USG is mandatory. 
For the menstrual disorders, hyperandrogenism to increase the sex hormone binding globulin, Oral contraceptives and metformin are the drug of choice. Although other insulin sensitizers like glitazones have equal effect, Metformin is the drug of choice because in case the patient becomes pregnant there is no teratogenic effect on foetus. The drug can be continued during pregnancy and it does not cause hypoglycaemia. It does not require the estimation of serum level of drug during the therapy.

The older treatment of wedge resection of the ovary is rarely needed as it causes adhesions and infertility. The latest laparoscopic ovarian drilling is done when there is a failure of medical treatment, in case of infertility cases. In PCOD the aetiology is obscure, but it is not without cure. It is a treatable disorder. By treating, PCOD a number of diseases like type 2 diabetes mellitus, ischemic heart disease, stroke, metabolic $\mathrm{X}$ syndrome, sleep apnoea, cancer breast, cancer endometrium and depression can be prevented.

Limitation of the study was that the sample size was small, and the diagnostic criteria also varies according the region.

\section{CONCLUSIONS}

Type 2 diabetes is seen in young individuals unlike yesteryears where Type 2 was noted only in elderly population. The type $2 \mathrm{DM}$ is seen in individuals who have risk factors like obesity, lack of exercise, carbohydrate diet and especially young PCOD women who attend infertility clinics with history of irregular menses and hirsutism. Such cases need regular screening to prevent the onset of Metabolic X syndrome, Type 2 DM, Stroke, IHD, CA Breast, CA endometrium, sleep apnoea, depression and bipolar disorders.

In order to prevent the sequelae of the PCOD, catch them young to change the lifestyle and diet, insist on exercise, and maintain the BMI which will prevent complications. In future regular screening should be offered by the government and also the private NGOs to provide free, regular screening for PCOD patients.

These patients are very young adults and need counseling, education, explanation, empathetic approach and support to deal with this disease causing complex multiple organ-system dysfunction.

\section{ACKNOWLEDGEMENT}

Authors thank the ethical committee of Karuna Medical College, staff of Department of OBG, Mr. Rahim (Manager), Dr. Vasanthamalai (The Principal) and Dr. Seema Devi Patil (The Vice Principal).

\section{REFERENCES}

[1] Naz MSG, Tehrani FR, Majd HA, et al. The prevalence of polycystic ovary syndrome in adolescents: a systematic review and meta-analysis. International Journal of Reproductive BioMedicine (Yazd) 2019;17(8):533-42.

[2] Pasquali R. Contemporary approaches to the management of polycystic ovary syndrome. Ther Adv Endocrinol Metab 2018;9(4):123-34.

[3] Kalra A, Nair S, Rai L. Association of obesity and insulin resistance with dyslipidemia in Indian women with polycystic ovarian syndrome. Indian J Med Sci 2006;60(11):447-53.

[4] Misra A, Chowbey P, Makkar BM, et al. Consensus statement for diagnosis of obesity, abdominal obesity and the metabolic syndrome for Asian Indians and recommendations for physical activity, medical and surgical management. J Assoc Physicians India 2009;57:163-70.

[5] Norman RJ, Mahabeer S, Masters S. Ethnic differences in insulin and glucose response to glucose between white and Indian women with polycystic ovary syndrome. Fertil Steril 1995;63(1):58-62.

[6] Kahn CR, Flier JS, Bar RS, et al. The syndromes of insulin resistance and acanthosis Nigricans. Insulin-receptor disorders in man. N Engl J Med 1976;294(14):739-45.

[7] Yao K, Bian C, Zhao X. Association of polycystic ovary syndrome with metabolic syndrome and gestational diabetes: aggravated complication of pregnancy. Exp Ther Med 2017;14(2):1271-6.

[8] Huang PL. A comprehensive definition for metabolic syndrome. Dis Model Mech 2009;2(5-6):231-7.

[9] Lorena I, Rasquin L, Mayrin JV. Polycystic Ovarian Disease (Stein-Leventhal Syndrome). Treasure Island (FL): StatPearls Publishing 2020.

[10] Rashidi H, Tehrani FR, Khomami MB, et al. To what extent does the use of the Rotterdam criteria affect the prevalence of polycystic ovary syndrome? A communitybased study from the Southwest of Iran. Eur J Obstet Gynecol Reprod Biol 2014;174:100-5.

[11] Witchel SF, Oberfield S, Rosenfield RL, et al. The diagnosis of polycystic ovary syndrome during adolescence. Horm Res Paediatr 2015;83:376-89.

[12] Yildiz BO, Bolour S, Woods K, et al. Visually scoring hirsutism. Hum Reprod Update 2010;16(1):51-64. 